\section{Microincision cataract surgery combined with vitrectomy: a case series}

\begin{abstract}
Aim The objective of this study was to present the results of combined phacovitrectomy using $1.8 \mathrm{~mm}$ microincision cataract surgery (MICS) with special emphasis on the anterior segment complications in this group.

Methods Retrospective, single-centre case series involving consecutive patients undergoing phacovitrectomy in a single centre in the United Kingdom during a 6-month period.

Results A total of 52 eyes underwent combined MICS and pars plana vitrectomy.

Intraoperative complications included posterior capsule rupture $(n=2)$, minor iris trauma during phacoemulsification $(n=1)$, iatrogenic retinal tears $(n=2)$, and entry site break $(n=1)$. Postoperatively two cases had significant inflammation, one of which resulted in $360^{\circ}$ posterior synaechiea, iris bombe, and raised intraocular pressure. Other complications included mild posterior synaechiae $(n=2)$, posterior capsular opacification $(n=3)$, cystoid macular oedema $(n=1)$, and hyphaema $(n=1)$, which spontaneously resolved. There were no cases of intraocular lens decentration. Two patients who underwent surgery for retinal detachment repair subsequently redetached. Among those having surgery for macular hole, non-closure was seen in one patient and one patient developed a retinal detachment.

Conclusion In conclusion, sub-2 mm MICS is a safe and effective technique in dealing with vitreoretinal disorders necessitating cataract surgery at the same time.

Eye (2014) 28, 386-389; doi:10.1038/eye.2013.300;

published online 10 January 2014

Keywords: MICS; phacovitrectomy; cataract; vitrectomy; phacoemulsification

Introduction

Both cataract and vitreoretinal surgery are evolving towards ever-smaller incisions. In retinal surgery, transconjunctival 'sutureless' pars plana vitrectomy with 23-, 25-, and 27-G techniques have been described. ${ }^{1-3}$ Likewise, microincision cataract surgery (MICS) can be achieved by sub- $2 \mathrm{~mm}$ incisions. ${ }^{4}$ The main advantages of MICS include minimising surgically induced astigmatism and reducing higher-order corneal aberrations. ${ }^{5}$ When performing a combined phacovitrectomy (sequential phacoemulsification/intraocular lens (IOL) insertion followed by pars plana vitrectomy; PPPV), a smaller corneal wound size may have additional advantages to the subsequent vitrectomy, including avoiding the need for wound suturing and preventing the anterior chamber from collapsing during the subsequent PPPV. We present a consecutive case series of patients undergoing combined phacovitrectomy using $1.8 \mathrm{~mm}$ (microincision) corneal wounds with special emphasis on anterior segment complications of the procedure.

\section{Materials and methods}

The authors present a single-centre, retrospective, consecutive case series. The medical records including electronic vitreoretinal surgical operation database of 52 cases of combined cataract surgery and vitrectomy operated from October 2011 to March 2012 were reviewed.

Preoperative data included patient's age, sex, best-corrected visual acuity, previous ocular history and indication for vitrectomy. Intraoperative data included corneal wound suturing if required, the type of IOL used and any complications.
\end{abstract}

Manchester Royal Eye Hospital, Central Manchester University Hospitals NHS Foundation Trust, Manchester Academic Health Science Centre and Institute of Human Development, University of Manchester, Manchester, UK

Correspondence:

N Patton, Manchester Royal Eye Hospital, Central Manchester University Hospitals NHS Foundation Trust, Manchester Academic Health Science Centre and Institute of Human Development, University of Manchester, Oxford Road, Manchester M13 9WH, UK

Tel: +44 (0)161 276 1234; Fax: +44 (0)161 2726618

E-mail: niall.patton@

cmft.nhs.uk

Received: 22 July 2013

Accepted in revised form:

14 November 2013

Published online:

10 January 2014
A Jalil, L Steeples, S Subramani, MS Bindra, F Dhawahir-Scala and N Patton 
Postoperative data included visual acuity, degree of anterior chamber inflammation, and intraocular pressure (IOP) measured by Goldman applanation tonometry. Anterior segment complications such as wound leak, IOL decentration and posterior capsule opacification, and posterior segment complications such as endophthalmitis and retinal detachment were noted. Significant inflammation was defined as inflammation needing an increase in topical steroids above the usual postoperative regimen.

\section{Surgical methods}

Cataract surgery was performed before vitrectomy, using a microincision technique, with a $1.8 \mathrm{~mm}$ clear corneal incision. The IOL was inserted without enlarging the wound using a wound-assisted technique, where the cartridge tip of the lens injector projected firmly into the wound without entering the anterior chamber. Akreos AO MI-60 Micro Incision Lens (Bausch and Lomb, Rochester, NY, USA) was used in all cases except where there was a posterior capsular rupture, where a larger MA60 lens (Alcon Laboratories Inc., Fort Worth, TX, USA) was implanted in the sulcus. If the anterior chamber was formed and stable at the end of cataract surgery, the main corneal wound was not sutured, and the surgeons proceeded to the vitrectomy using the Stellaris PC Vitrectomy System (Bausch and Lomb). The vitrectomies carried out in the department had a standard approach comprising core vitrectomy and peripheral shave anterior to the equator without indentation except for retinal detachments, where a complete peripheral shave with indentation was carried out. All cases underwent an internal search to identify iatrogenic anterior retinal tears and sclerotomy-associated retinal tears towards the end of the procedure, and if present these were treated accordingly.

Patients were given prednisolone acetate $1 \%$ eye drops 2 hourly for 1 week and four times for 3 more weeks, and topical antibiotics and cycloplegics for 2 weeks.

All cases were routinely seen on day 1, 2 weeks, 6-8 weeks, and then 3 months postoperatively with variations at surgeon's discretion.

\section{Results}

A total of 52 cases (52 eyes) underwent combined phacoemulsification plus insertion of IOL and PPPV. The average patient age was 69 years (range $47-87$ years) with 23 female subjects and 29 male subjects.

The minimum follow-up period was 8 weeks. Two patients were discharged from the service at this point, a further patient requested follow-up in their local unit, and one patient did not attend beyond the 8-week review.
All other patients were followed up for longer. The mean follow-up was 19 weeks (range 8-60 weeks).

The underlying diagnoses of all these cases are given in Table 1.

Preoperatively the visual acuities ranged from 0.4 $\log$ MAR to perception of light. In 15 patients, the recorded visual acuity was count fingers or worse. Among those with visual acuity better than or equal to $1.0 \log \mathrm{MAR}$, the average acuity was $0.8 \log \mathrm{MAR}$ with $0.4-0.6$ in 7 cases, $0.7-0.9$ in 20 cases, and 1.0 in 10 cases.

The surgery was performed by two Consultant Vitreoretinal Surgeons $(n=35)$ and two Vitreoretinal Fellows under consultant supervision $(n=17)$. Corneal sutures (10-0 nylon) were placed in 11 of 52 eyes mostly in the initial few surgeries where there was a learning curve for wound-assisted injection and also suspicion regarding the stability of anterior chamber during subsequent vitrectomy. Akreos AO MI-60 lens (Bausch and Lomb) was implanted in the capsular bag in 51 cases, and in all these cases the wound was not enlarged. MA60 lens was placed in the sulcus in the remaining case owing to posterior capsule rupture, and in this case the wound had to be extended to introduce a larger lens. 23-G PPPV was performed in 43 cases, of which 4 required suturing of one or more ports. The remaining nine cases had 20-G vitrectomy because of surgeon's preference or alternative case requirements.

Intraoperative complications reported were posterior capsule rupture $(n=2)$, minor iris trauma during phacoemulsification $(n=1)$, iatrogenic retinal tears $(n=2)$, entry site break $(n=1)$, and surgery abandoned because of inoperable tractional retinal detachment in one case.

On postoperative day 1 all corneal wounds were secure with no leak. Two cases had significant inflammation ( $>2+$ anterior chamber cells) noted at 2 weeks, one of which developed $360^{\circ}$ posterior synaechiea, iris bombe, and raised IOP, and subsequently redetached 3 months later. One of these patients had iris

Table 1 Underlying diagnoses of all cases requiring phacovitrectomy

\begin{tabular}{lc}
\hline Underlying diagnosis & \\
\hline ERM & 11 \\
Idiopathic macular hole & 15 \\
RD & 8 \\
Diabetic TRD & 3 \\
Diabetic vitreous haemorrhage & 4 \\
Vitreal haemorrhage-other & 3 \\
VMT & 5 \\
Posterior uveitis & 3 \\
Total & 52
\end{tabular}

Abbreviations: ERM, epiretinal membrane; RD, retinal detachment; TRD, tractional retinal detachment; VMT, vitreomacular traction. 
trauma preoperatively, whereas no identifiable risk factor was found in the other patient. In total, posterior synaechiae developed in three cases and were $<2$ clock hours in the other two cases. Hyphaema was noted in one patient, which cleared spontaneously.

During the follow-up, there have been no cases of IOL decentration or capsule phimosis, and three patients had developed posterior capsule opacification. Postoperative cystoid macular oedema developed in one patient where vitrectomy was carried out for posterior uveitis.

Two patients out of eight who underwent retinal detachment repair subsequently redetached (both were re-do surgeries). Among the macular hole subgroup, non-closure was seen in one patient who declined further surgery and one patient developed a retinal detachment; the success rate was comparable with previous reports. ${ }^{6}$

Postoperatively visual acuity improved in 46/52 cases and remained unchanged in two cases. The average postoperative visual acuity (excluding three cases worse than $1.0 \log$ MAR from analysis) was $0.5 \log$ MAR.

The average improvement in visual acuity was 0.3 $\log$ MAR. The range of improvement was from 2 letters to $>0.9 \log$ MAR with an improvement from HM to 0.1 $\log$ MAR. Visual acuity worsened in four cases from the preoperative level and the aetiology was inoperable detachment $(n=1)$, diabetic maculopathy $(n=2)$, and persistent retinal thickening after ERM peel $(n=1)$. In three of these patients, the preoperative visual acuity was $0.8 \log$ MAR or worse and worse than $1.0 \log$ MAR postoperatively.

\section{Discussion}

The most common complication of vitrectomy is progression of nuclear sclerotic cataract, especially in the elderly population. Furthermore, cataract surgery in a vitrectomised eye can be challenging with fluctuating anterior chamber, weak zonules, and unstable posterior capsular support. ${ }^{7}$ Hence, there has been an increasing trend towards combining cataract surgery with immediate sequential PPPV for a variety of indications such as macular holes, retinal detachment, and proliferative diabetic retinopathy. ${ }^{7-9}$ A combined PPPV is considered a safe approach with faster rehabilitation, which prevents the need for a second operation for a postvitrectomy cataract. The fact that vitrectomy is increasingly small gauge, sutureless, and hence quicker is also contributing to the popularity of combined phacovitrectomy.

When performing PPPV, IOL has been traditionally implanted through a $3 \mathrm{~mm}$ corneal wound, either at the completion of phacoemulsification before starting vitrectomy or at the end of phacovitrectomy and insertion of internal tamponade. ${ }^{10,11}$ The results of MICS $(1.8 \mathrm{~mm})$ in combination with PPPV have not been presented to our knowledge before. In our study, the IOL was always implanted before proceeding to vitrectomy. No difficulty was encountered either in complex dissections over posterior pole or in peripheral shave of the vitreous because of the prismatic effect of IOL edge. In addition, any indentation during the posterior segment surgery did not compromise the stability of anterior chamber because of the stability of MICS wound.

The most important anterior segment intraoperative complication seen in our patients was the posterior capsular rupture in 2 out of 52 patients (3.8\%). This is comparable to the $4.2 \%$ posterior capsular breach seen in a similar series of 72 eyes by Krishnan et al, ${ }^{11}$ which only looked at PPPV for macular holes. When looking at cataract surgery in vitrectomised eyes, Pardo-Munoz et $a l^{12}$ found a posterior capsular tear rate of $4 \%$ and zonular dialysis of $5 \%$, a much higher complication rate as compared with our series.

Demetriades et $a l^{7}$ looked at 122 cases where PPPV was carried out for various vitreoretinal pathologies, and concluded that the main postoperative anterior segment complications were inflammation and posterior capsular opacification (PCO), corneal epithelial defects, IOP rise, and IOL dislocation with iris capture. They recommended smaller curvilinear capsulorhexis, the in-the-bag placement of IOLs with larger optics, suturing of cataract wounds before vitrectomy, and the avoidance of long-acting dilating drops in patients with intravitreal gas. In our series, MICS was carried out with a $1.8 \mathrm{~mm}$ corneal wound, mostly avoiding the need for a corneal suture before vitrectomy. The IOL has four haptics, which ensured a completely stable lens in the bag reducing the chances of lens dislocation and iris capture. No cases of IOL decentration were noted in our cohort during the follow-up period.

When cataract surgery is combined with vitrectomy, there is a greater likelihood of increased postoperative inflammation due to increased surgical time, intraoperative manipulation, and the use of internal tamponade, especially silicone oil. A strong fibrinous inflammatory response is especially seen in patients with diabetes, which may necessitate the use of intensive topical steroids. ${ }^{9}$ We had a severe inflammatory response in only two cases $(3.8 \%)$, one of which ended up with $360^{\circ}$ posterior synaechiae and iris bombe. This is much less than the rate of severe inflammation of up to $6-7 \%$ reported in some of the other studies, 10,13 and may be due to a more stable anterior chamber when MICS precedes vitrectomy.

PCO is an important complication of a combined phacovitrectomy, its incidence varying from $10 \%$ to as high as $51.1 \% .{ }^{14,15} \mathrm{~A}$ number of factors contribute to PCO formation after PPPV, including increased surgical manipulation and inflammation, the use of long-acting 
gas tamponade, and postoperative posturing. ${ }^{16}$ Also, it has been shown by Iwase et al ${ }^{14}$ that PCO rates are lower following a transconjunctival 23-G phacovitrectomy when compared with 20-G phacovitrectomy. Our series had a PCO rate of $5.8 \%$, but our follow-up period (mean of 4 months) is too short to compare it with some of the studies with higher PCO rates.

Regarding other anterior segment complications, we only had one case of hyphaema, which resolved spontaneously in a few days, one case of significant IOP rise due to iris bombe, and no case with corneal oedema. This is significantly less than the rates reported by many of the other studies, especially where phacoemulsification was combined with $20-\mathrm{G}$ vitrectomy. ${ }^{7}$ A reason for this may be that there are less chances of a raised IOP in sutureless tranconjunctival vitrectomy, with the cornea more likely to retain clarity due to less intraoperative trauma and less IOP spikes. Krishnan et al ${ }^{11}$ have shown that postoperative IOP is significantly higher after 20-G phacovitrectomy compared with 23-G PPPV at day 1 and just below significance at day 7. Most of our cases were 23-G PPPV, which might explain our lower rates of IOP spike in the immediate postoperative period.

In conclusion, we present a series of consecutive patients who had sub-2 mm MICS followed by a vitrectomy. Our small study suggests that this is safe and efficient technique in dealing with cataracts in the presence of vitreoretinal pathology.

\section{Summary}

What was known before

- A combined phacovitrectomy leads to a faster rehabilitation, preventing the need for a second operation for a postvitrectomy cataract.

- When performing this procedure, IOL has been traditionally implanted through a $3 \mathrm{~mm}$ corneal wound.

\section{What this study adds}

- We present the results of MICS $(1.8 \mathrm{~mm})$ in combination with vitrectomy, which have not been presented to our knowledge before.

- Our results show that this is safe and efficient technique in dealing with cataracts in the presence of vitreoretinal pathology.

\section{Conflict of interest}

The authors declare no conflict of interest.

\section{References}

1 Fujii GY, de Juan Jr E, Humayun MS, Chang TS, Pieramici DJ, Barnes A et al. Initial experience using the transconjunctival sutureless vitrectomy system for vitreoretinal surgery. Ophthalmology 2002; 109: 1814-1820.

2 Eckardt C. Transconjunctival sutureless 23-gauge vitrectomy. Retina 2005; 25: 208-211.

3 Oshima Y, Wakabayashi T, Sato T, Ohji M, Tano Y. A 27-gauge instrument system for transconjunctival sutureless microincision vitrectomy surgery. Ophthalmology 2010; 117: 93-102.

4 Kim EC, Byun YS, Kim MS. Microincision versus small-incision coaxial cataract surgery using different power modes for hard nuclear cataract. J Cataract Refract Surg 2011; 37: 1799-1805.

5 Denoyer A, Denoyer L, Marotte D, Georget M, Pisella PJ. Intraindividual comparative study of corneal and ocular wavefront aberrations after biaxial microincision versus coaxial small-incision cataract surgery. Br J Ophthalmol 2008; 92: 1679-1684.

6 Ezra E, Gregor ZJ. Surgery for idiopathic full-thickness macular hole: two-year results of a randomized clinical trial comparing natural history, vitrectomy, and vitrectomy plus autologous serum: Morfields Macular Hole Study Group Report no. 1. Arch Ophthalmol 2004; 122(2): 224-236.

7 Demetriades AM, Gottsch JD, Thomsen R, Azab A, Stark WJ, Campochiaro PA et al. Combined phacoemulsification, intraocular lens implantation, and vitrectomy for eyes with coexisting cataract and vitreoretinal pathology. Am J Ophthalmol 2003; 135: 291-296.

8 Dhawahir-Scala FE, Maino A, Saha K, Mokashi AA, McLauchlan R, Charles S. To posture or not to posture after macular hole surgery. Retina 2008; 28: 60-65.

9 Lahey JM, Francis RR, Kearney JJ. Combining phacoemulsification with pars plana vitrectomy in patients with proliferative diabetic retinopathy: a series of 223 cases. Ophthalmology 2003; 110: 1335-1339.

10 Zheng QX, Wu RH, Zhang YP, Xu M, Li WS. Anterior segment complications after phacoemulsification combined vitrectomy and foldable intraocular lens implantation. Int J Ophthalmol 2010; 3: 249-254.

11 Krishnan R, Tossounis C, Fung Yang Y. 20-Gauge and 23-gauge phacovitrectomy for idiopathic macular holes: comparison of complications and long-term outcomes. Eye (Lond) 2013; 27: 72-77.

12 Pardo-Munoz A, Muriel-Herrero A, Abraira V, Muriel A, Muñoz-Negrete FJ, Murube J. Phacoemulsification in previously vitrectomized patients: an analysis of the surgical results in 100 eyes as well as the factors contributing to the cataract formation. Eur J Ophthalmol 2006; 16: 52-59.

13 Wensheng L, Wu R, Wang X, Xu M, Sun G, Sun C. Clinical complications of combined phacoemulsification and vitrectomy for eyes with coexisting cataract and vitreoretinal diseases. Eur J Ophthalmol 2009; 19: 37-45.

14 Iwase T, Oveson BC, Nishi Y. Posterior capsule opacification following 20- and 23-gauge phacovitrectomy (posterior capsule opacification following phacovitrectomy). Eye (Lond) 2012; 26: 1459-1464.

15 Ling R, Simcock P, McCoombes J, Shaw S. Presbyopic phacovitrectomy. Br J Ophthalmol 2003; 87: 1333-1335.

16 Rahman R, Briffa BV, Gupta A, Chinn DJ. Factors contributing to posterior capsule opacification following 23-gauge transconjunctival phacovitrectomy. Ophthalmic Surg Lasers Imag 2011; 42: 229-233. 